\title{
Three-dimensional morphological analysis of true acetabulum in Crowe type IV hip dysplasia via standard-sized cup-simulated implantation
}

\author{
Yuhui Yang ${ }^{1 \# \wedge}$, Yuanchen $\mathrm{Ma}^{1 \#}$, Qingtian $\mathrm{Li}^{1}$, Bofu Lin ${ }^{1}$, Hang Dong ${ }^{2} \wedge$, Qiujian Zheng ${ }^{1,3}$ \\ ${ }^{1}$ Department of Orthopedics, Guangdong Provincial People's Hospital, Guangdong Academy of Medical Sciences, Guangzhou, China; ${ }^{2}$ Department \\ of Orthopedics, China-Japan Union Hospital of Jilin University, Changchun, China; ${ }^{3}$ The Second School of Clinical Medicine, Southern Medical \\ University, Guangzhou, China
}

Contributions: (I) Conception and design: Y Yang, H Dong, Q Zheng; (II) Administrative support: All authors; (III) Provision of study materials or patients: Y Yang, Y Ma, Q Li; (IV) Collection and assembly of data: Y Yang, B Lin; (V) Data analysis and interpretation: All authors; (VI) Manuscript writing: All authors; (VII) Final approval of manuscript: All authors.

"These authors contributed equally to this work.

Correspondence to: Hang Dong. Department of Orthopedics, China-Japan Union Hospital of Jilin University, Changchun 130033, China. Email: donghanghappy@163.com; Qiujian Zheng. Department of Orthopedics, Guangdong Provincial People's Hospital, Guangdong Academy of Medical Sciences, Guangzhou 510080, China. Email: zhengqiujian@gdph.org.cn.

Background: Acetabular reconstruction in Crowe type IV developmental dysplasia of the hip (DDH) can be a challenging procedure for surgeons. A high risk of acetabular revision has been reported to be associated with extremely small acetabular prostheses. However, to our knowledge, quantitative morphological and coverage evaluations of the true acetabulum in Crowe IV hips have been infrequently conducted. Useful bony landmarks for acetabular reconstruction can also facilitate satisfactory intraoperative implantation. The aim of the present study was to investigate the anatomical size, orientation angles, and 2/3-dimensional $(2 \mathrm{D} / 3 \mathrm{D})$ coverage parameters of the true acetabulum in Crowe IV hips; evaluate the feasibility of standard cup ( $>44 \mathrm{~mm}$ ) implantation at the true acetabulum in Crowe IV hips; and identify the optimal position and useful bony landmarks of the acetabular reaming center in Crowe IV hips.

Methods: A total of 42 Crowe IV hips in 37 patients and 36 normal hips were included in this study. Based on pelvic 3D computed tomography $(\mathrm{CT})$ reconstruction, anatomical size and integral volume of the true acetabulum were measured quantitatively. Through standard-size cup-simulated implantation, morphological assessments of the true acetabulum included Cup-CE, Cup-Sharp, acetabular anteversion angle, and thickness of the medial wall. Acetabular sector angles (ASAs) and the component coverage ratio were measured to provide coverage indices. Acetabular reconstruction was also performed at different vertical levels to measure medial bone stock and 3D component coverage. Bony landmarks for optimal component center location were also determined.

Results: The anatomic shape and volume of the acetabular triangle were significantly smaller in Crowe IV hips. Compared with the control group, the dysplastic acetabulum was more anteverted and abductive, with a thicker medial wall. According to the true acetabulum, bone stock was relatively sufficient in the posterior direction and prominently deficient in the anterosuperior and superior direction. The average 3D component coverage reached $79.89 \%$ by standard-sized cup implantation, with the most satisfactory coverage achieved at the true acetabulum (at the level of $13.32 \mathrm{~mm}$ above the transverse acetabular ligament). Regarding the component opening plane, the optimal component center was located at the midpoint

^ ORCID: Yuhui Yang, 0000-0002-1938-0635; Hang Dong, 0000-0001-6584-371X. 
between the superolateral and posteroinferior points of the true acetabulum.

Conclusions: The most satisfactory coverage was achieved at the level of the true acetabulum, of which the most prominent deficiency was mainly located in the anterosuperior and superior directions. The optimal component center was determined to be the midpoint between the superolateral and posteroinferior points of the true acetabulum.

Keywords: Crowe type IV developmental dysplasia of the hip (DDH); simulated implantation; morphological assessment; 3-dimensional (3D) coverage; bony landmark

Submitted Aug 13, 2021. Accepted for publication Feb 05, 2022.

doi: 10.21037/qims-21-803

View this article at: https://dx.doi.org/10.21037/qims-21-803

\section{Introduction}

Total hip arthroplasty (THA) in patients with high-riding Crowe type IV developmental dysplasia of the hip (DDH) is a challenging procedure for surgeons. It is crucial for the restoration of anatomical joint biomechanics and reconstruction of the center of rotation (1-3). For acetabular reconstruction, challenges associated with managing the true acetabulum include a distinctively triangular socket, remarkably increased anteversion, hypoplastic anterosuperior wall, and decreased bone stock (4-6).

In terms of severe dysplasia of the hip, the use of a small acetabular prosthesis is recommended to achieve satisfactory bony coverage $(7,8)$. However, polyethylene erosion and risk of postoperative dislocation have been associated with small prosthesis size (9-11). Follow-up studies have shown that the risk of acetabular revision was significantly higher than that of femoral revision $(9,11)$. During prior surgeries, we noted that standard size cup $(>44 \mathrm{~mm})$ can also achieve acceptable implant coverage by careful 3-dimensional (3D) preoperative planning and accurate acetabular reaming. Zhou et al. (12) claimed that a standard cup was feasible when implanted posteriorly and inferiorly, resulting in promising bony coverage and clinical survival. In their studies, Hartofilakidis et al. and $\mathrm{Xu}$ et al. $(7,13)$ recommended a superior and posterior standardsized reaming for relatively sufficient bone stock, which was similar to the findings of our previous study (4). However, relevant research involving quantitative coverage status and ideal reaming location are still limited for standard-sized cup implantation.

Few studies have addressed the morphological evaluation of true acetabulum reconstruction and optimal bony landmarks for standard-sized cup implantation in Crowe IV hips $(5,14)$. Using a 3D implantation simulation method, the aim of the present study was to investigate the anatomical size, orientation angles, and 2-dimensional (2D)/3D coverage parameters of the true acetabulum in Crowe IV hips; evaluate the feasibility of standard cup $(>44 \mathrm{~mm}$ ) implantation at the true acetabulum in Crowe IV hips; and identify the optimal position and useful bony landmarks of acetabular reaming center in Crowe IV hips.

We present the following article in accordance with the Materials Design Analysis Reporting (MDAR) checklist (available at https://qims.amegroups.com/article/ view/10.21037/qims-21-803/rc).

\section{Methods}

\section{Study design and setting}

This study was conducted in accordance with the Declaration of Helsinki (as revised in 2013). The study was approved by the Institutional Review Board of Guangdong Provincial People's Hospital (No. 2019528HR1), and informed consent was provided in writing by all participants. We retrospectively reviewed the preoperative imaging data of 352 patients (496 hips) with DDH who were admitted to our institution from January 2010 to January 2019. According to the Crowe classification, 46 participants (58 hips) were graded as type IV DDH on standing anteroposterior pelvic radiographs. Of those 46, 7 (12 hips) cases who had substandard scans and 2 (4 hips) who had undergone previous surgery were excluded. Therefore, 42 dysplastic hips in 37 cases met the inclusion criteria and were retrospectively evaluated. A total of 18 patients (36 hips) without acetabular fracture or deformities who had undergone computed tomography (CT) angiography to diagnose proximal femoral fractures were selected as controls. Demographic data for the participants are shown in Table 1. 
Table 1 Demographic data of participants

\begin{tabular}{lcc}
\hline Characteristics & Normal & Crowe IV DDH \\
\hline Hips/patients $(\mathrm{n})$ & $36 / 18$ & $42 / 37$ \\
Male/female $(\mathrm{n})$ & $6 / 30$ & $5 / 32$ \\
Age (years) & $39.00 \pm 10.13[20-54]$ & $42.73 \pm 13.62(21-70)$ \\
Height $(\mathrm{cm})$ & $163.17 \pm 7.40[155-185]$ & $157.50 \pm 10.22(141-175)^{\star}$ \\
Weight $(\mathrm{kg})$ & $64.72 \pm 7.82[49-87]$ & $56.19 \pm 11.12(33-84)^{\star *}$ \\
Body mass index $\left(\mathrm{kg} / \mathrm{m}^{2}\right)$ & $24.34 \pm 2.72[18.00-29.14]$ & $18.75 \pm 9.97(21.66-35.42)^{\star *}$ \\
\hline
\end{tabular}

Values are expressed as mean \pm standard deviation [range]. * $\mathrm{P}<0.05 ;{ }^{* \star}, \mathrm{P}<0.01$ when compared with the control group. DDH, developmental dysplasia of the hip.

\section{$3 D$ reconstruction and acetabular size analysis}

Pelvic CT was performed with a Toshiba Aquilion CT scanner $(120 \mathrm{kVp}, 320 \mathrm{Ma}, 512 \times 512$ matrix; slice thickness: $0.5 \mathrm{~mm}$; Toshiba, Otawara, Japan) at the Guangdong Provincial People's Hospital. Participants were placed in a neutral supine position with their patellae facing the ceiling. Scanning was performed from the iliac crest to the distal third of the femur. All standard CT slices were saved in Digital Imaging and Communications in Medicine (DICOM) format and imported into Mimics 19.0 software (Materialise, Leuven, Belgium) for 3D reconstruction. Before simulation and measurement, the pelvic position was standardized with reference to the anterior pelvic plane coordinate system $(4,15)$, determined by the anterior superior iliac spines (ASIS) and the pubic tubercles bilaterally (Figure 1A). According to resliced CT images, the $3 \mathrm{D}$, coronal, sagittal, and transverse views were presented simultaneously in Mimics software. Morphology and bone stock distribution of the true acetabulum were compared between Crowe IV hips and normal hips. According to the true acetabulum, the distal part of the cotyloid notch (DPCN) $(4,16,17)$, the most superolateral point, and the midpoint between them were digitized (Figure 1B). In the coronal and axial plane passing through the midpoint, acetabular length, height, width, and depth were measured to determine the acetabular size in the control and Crowe IV DDH group. According to the acetabular rim, the acetabular volume was also evaluated with reference to the acetabular opening plane $(18,19)$ (Figure 1C-1E).

\section{Simulating implantation of the acetabular component}

A set of hemispherical virtual acetabular components with negligible thickness was created by using 3-matic 9.0 software (Materialise). According to the standard size of on-shelf acetabular implants, the outer diameters of these egg-shell cups ranged from 44 to $60 \mathrm{~mm}$ in $2 \mathrm{~mm}$ intervals. These 3D models were imported into Mimics software in stereolithography (STL) format.

Based on the method described by Yang et al. and Sariali et al. $(4,16)$, the simulated acetabular replacement was performed by placing the component in the true acetabulum, oriented at $40^{\circ}$ abduction and $20^{\circ}$ anteversion. The cup size was chosen to best accommodate the anteroposterior diameter of the true acetabulum, which tended to utilize the osseous peak of the anterior bone columns in case of deficient bone stock. The inferior edge of the virtual cup was placed at the level of the DPCN, which was considered the position of the transverse acetabular ligament $(16,17)$. In the Crowe IV DDH group, the outer wall of the component was tangent to, but did not penetrate, the inner cortex of the medial acetabular wall to achieve the theoretical maximum coverage. In the control group, the outer wall of the component was tangent to the cortical bone edge of the cotyloid notch.

\section{Evaluations and measurements}

Based on the implantation simulation, the morphological assessments and coverage parameters included the following: (I) measurement of the Cup-CE angle and CupSharp angle (Figure 2A); (II) measurement of the acetabular anteversion angle and minimum thickness of medial acetabular wall (Figure 2B); (III) acetabular sector angles (ASAs). Based on the contact point between native bone and the component, we measured the anterior and posterior ASAs in the axial plane (Figure 2B). Further, the ASAs in 

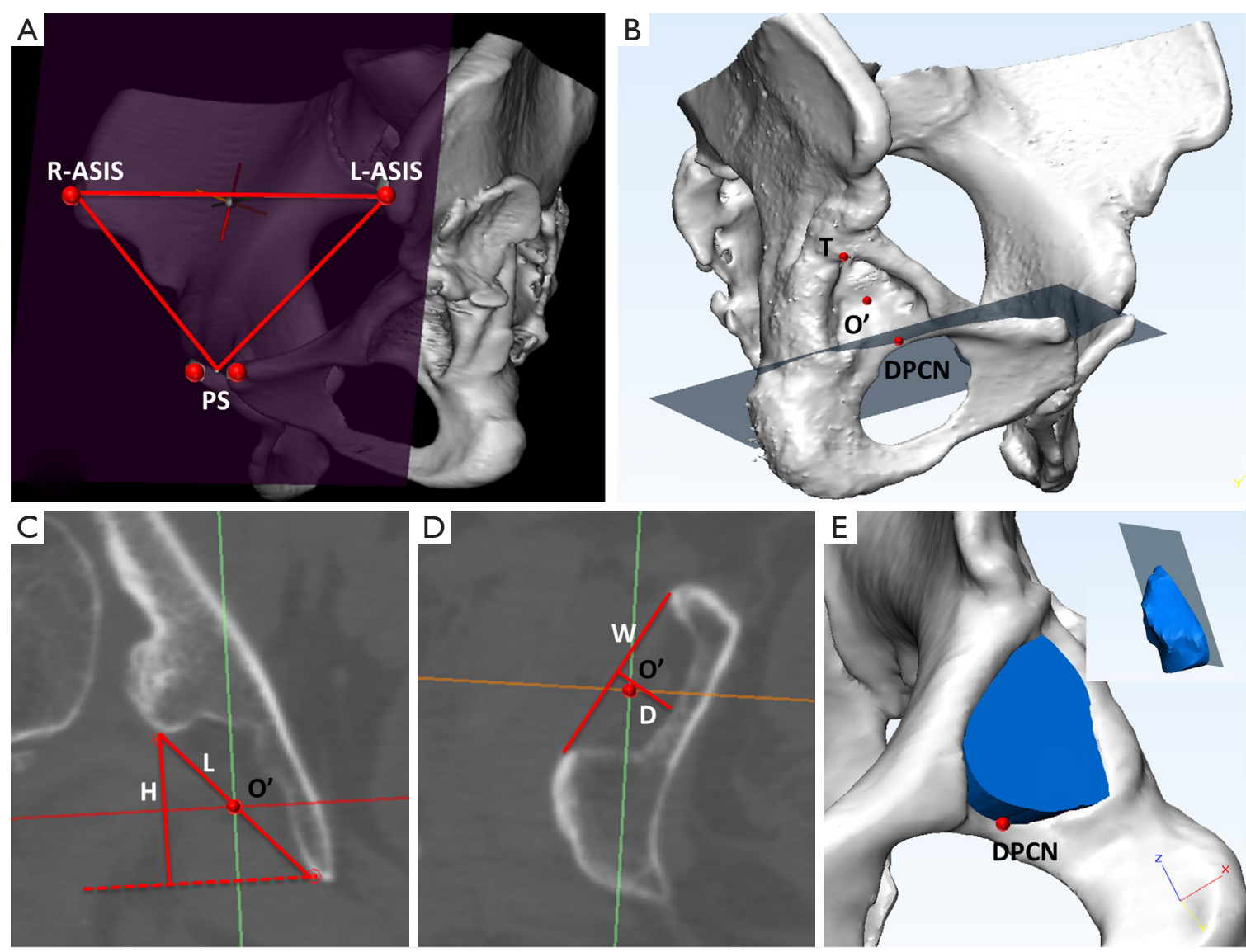

Figure $13 \mathrm{D}$ reconstruction and acetabular size analysis. (A) Anterior pelvic plane, left and right ASIS, and pubic tubercle. (B) Anatomical landmarks: DPCN, T, and midpoint of T and DPCN. (C) Coronal image. Acetabular height and acetabular length. (D) Axial image. Acetabular width and acetabular depth. (E) Acetabular volume analysis. ASIS, anterior superior iliac spines; DPCN, distal part of the cotyloid notch; T, most superolateral point of the acetabulum.

the $45^{\circ}$ anterosuperior direction, superior direction, and $45^{\circ}$ posterosuperior direction were also measured on the corresponding planes. Angles were respectively named A-ASA, P-ASA, AS-ASA, S-ASA, and PS-ASA (Figure 2C); (IV) $3 \mathrm{D}$ component coverage ratio. Using the simulation and Boolean function of Mimics, segmentations were performed according to the border between the covered and uncovered parts of the virtual cup (Figure 2D). Accordingly, the coverage was calculated as the ratio between the covered and total surface area; and $(\mathrm{V})$ the vertical height of the component center ( $\mathrm{V}-\mathrm{HCC}$ ) was defined as the vertical distance from the center of the component to the DPCN (Figure $3 A$ ). In Crowe type IV hips, implantation simulation with the same orientation was performed by placing the component at the initial preset position, with a $\mathrm{V}-\mathrm{HCC}=9 \mathrm{~mm}$. Subsequently, the virtual cup was stepwise elevated proximally by $3 \mathrm{~mm}$ increments, ranging from 9 to $39 \mathrm{~mm}$ (Figure 3B). At each level, the minimum thickness of the medial acetabular wall in the axial plane and the $3 \mathrm{D}$ component coverage ratio were evaluated.

\section{Location analysis of the acetabular component center}

On the basis of the implantation at the true acetabulum, the component opening plane was defined as the coordinate system (oriented at $40^{\circ}$ abduction and $20^{\circ}$ anteversion), with the origin positioned at the center of the acetabular component. Representative bone landmarks included the ipsilateral ASIS; the DPCN; the most superolateral point, the most anteroinferior point, and the most posteroinferior point of the true acetabulum rim were digitized (Figure 4A). Subsequently, the $3 \mathrm{D}$ pelvis model and acetabular 

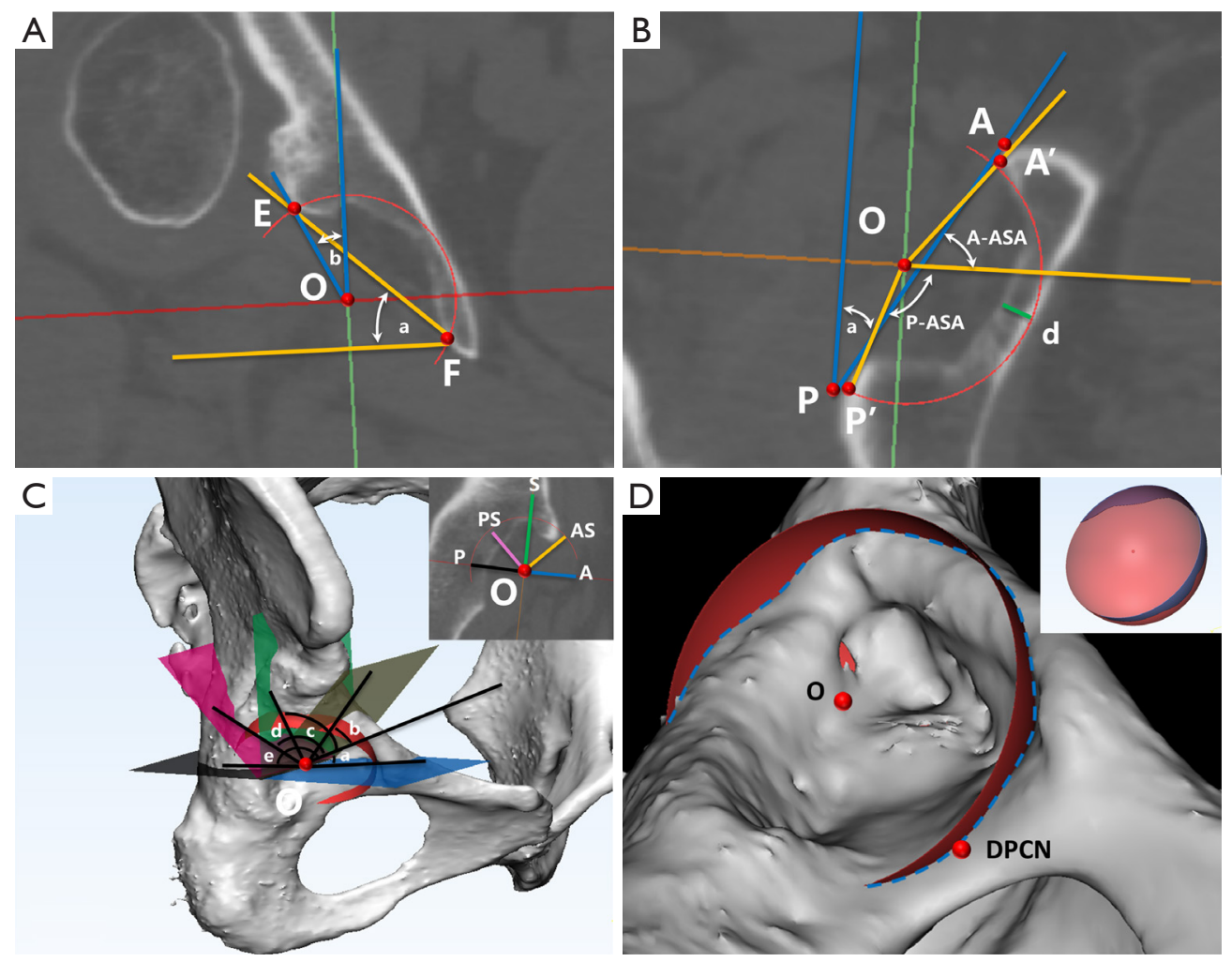

Figure 2 Morphological and coverage parameters analysis after implantation. (A) Cup-Sharp angle (a) and Cup-CE angle (b), measured with the coronal plane passing through the component center. Point $\mathrm{O}$ is the component center, points $\mathrm{E}$ and $\mathrm{F}$ are the superolateral and inferomedial borders between the component and the true acetabulum. (B) Acetabular anteversion angle (a), anterior and posterior ASAs and thickness of the medial acetabular wall (d), measured with the axial plane passing through the component center. Point $\mathrm{O}$ is the component center, points $\mathrm{A}$ and $\mathrm{P}$ are the anterior and posterior borders of the true acetabulum, points $\mathrm{A}^{\prime}$ and $\mathrm{P}^{\prime}$ are the anterior and posterior borders between the component and the true acetabulum. Direction of the medial wall thickness measurement is perpendicular to the acetabular opening (line AP). (C) ASAs through the component center (point O) were measured in the following 5 directions: anterior (a), anterosuperior (b), superior (c), posterosuperior (d), and posterior (e). (D) Coverage ratio of the acetabular component was calculated according to the covered surface area and the total surface area. O is the component center. ASAs, acetabular sector angles; DPCN, distal part of the cotyloid notch.

component were imported into 3-matic software. Based on the coordinate system, bony landmarks were projected to the component plane to characterize the distributed regularity and the location of the component center (Figure 4B).

\section{Statistical analysis}

To assess interobserver reliability, 2 experienced surgeons (YY and YM) performed the simulating implantation, point selection, and corresponding measurements independently. To assess intraobserver reliability, implantation, points selection, and measurements were removed and repeated twice at monthly intervals by YY. Intraclass correlation coefficient (ICC) was used to calculate interobserver and intraobserver effects.

All statistical analyses were performed using SPSS version 21.0 (IBM Corp., Armonk, NY, USA). A posthoc power calculation was determined by the statistical power analyses G Power 3.1 to eliminate type II error (20). Group comparisons for quantitative data were performed using unpaired Student's $t$-tests, and categorical data were compared using the $\chi^{2}$-test. Correlations between 2 continuous indices were analyzed using Spearman's correlation coefficient. Paired $t$-test was used to compare the difference of 2 selected landmark distances towards the component center. A P value $<0.05$ was considered 

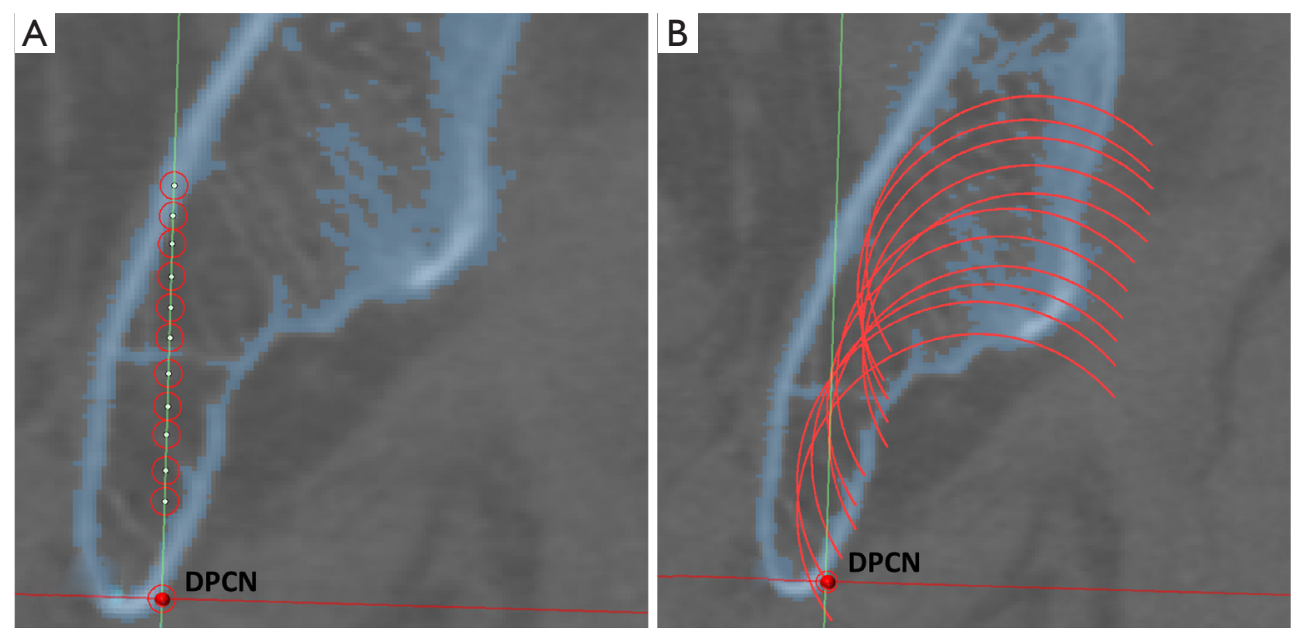

Figure 3 Acetabular reconstruction at different vertical levels. (A) Different vertical levels from the DPCN are shown. (B) Acetabular reconstruction was performed at different vertical levels. DPCN, distal part of the cotyloid notch.
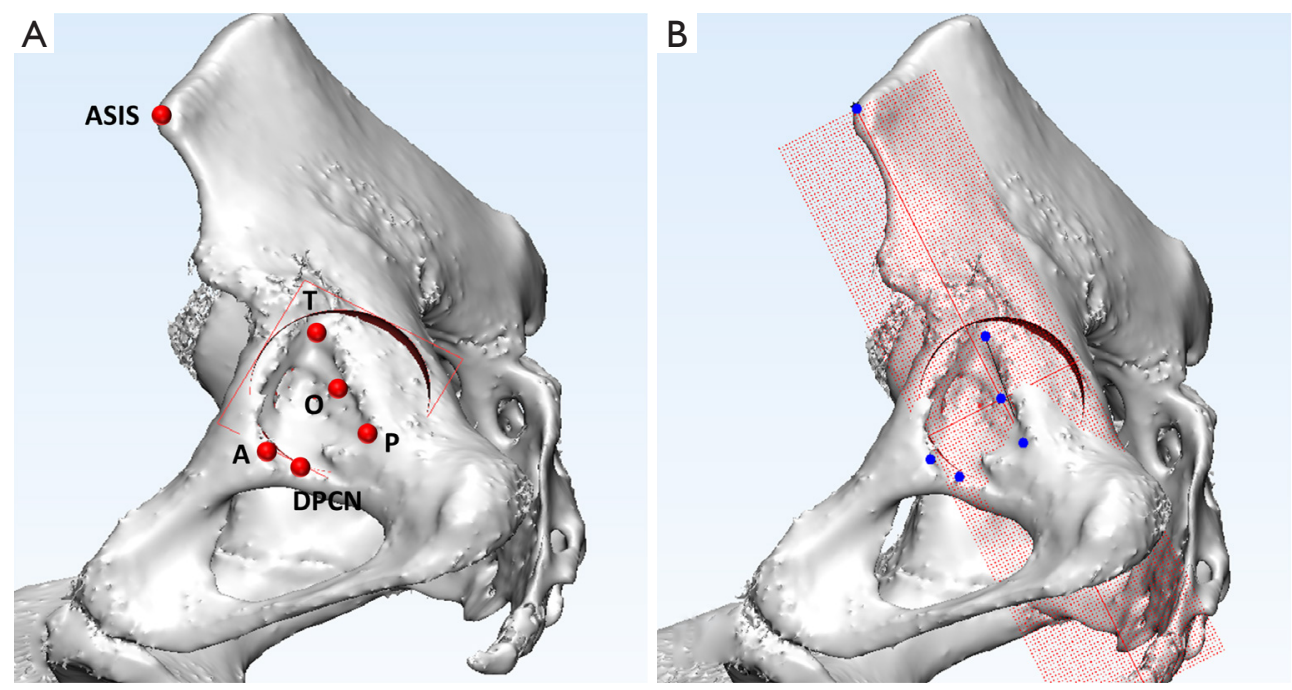

Figure 4 Location analysis of the acetabular component center. (A) Representative bone references are shown. ASIS; DPCN; points T, A, and $\mathrm{P}$ indicate the most superolateral, anteroinferior, and posteroinferior point of the true acetabulum, and $\mathrm{O}$ is the component center. (B) Based on the coordinate system, bone references are projected and marked as blue points. ASIS, anterior superior iliac spines; DPCN, distal part of the cotyloid notch.

statistically significant.

\section{Results}

\section{Acetabular anatomy and morphological analysis}

Compared with the hemispherical shape of the normal acetabulum, the true acetabulum in Crowe IV hips tended to be markedly triangular and shallow. As detailed in Table 2, all aspects of the anatomic parameter in Crowe IV hips were significantly smaller than in normal hips, especially the acetabular width $(25.41 \pm 5.37$ vs. $50.14 \pm 2.48 \mathrm{~mm})$. The accurate volume of the dysplastic socket was less than one-third of the normal acetabulum. Both the size and vertical height of the chosen component were significantly greater in normal hips. At the level of the acetabular component center, dysplastic acetabula were found to be 
Table 2 Acetabular anatomy and morphological parameters after implantation

\begin{tabular}{lcc}
\hline Parameters & Normal $(\mathrm{n}=36)$ & Crowe IV DDH $(\mathrm{n}=42)$ \\
\hline Acetabular length $(\mathrm{mm})$ & $54.49 \pm 2.05(51.37-59.12)$ & $39.74 \pm 4.54(30.53-50.65)^{\star \star}$ \\
Acetabular height $(\mathrm{mm})$ & $34.10 \pm 3.00(28.20-42.40)$ & $29.59 \pm 4.37(19.41-38.27)^{\star \star}$ \\
Acetabular width $(\mathrm{mm})$ & $50.14 \pm 2.48(46.74-54.90)$ & $25.41 \pm 5.37(15.38-37.33)^{\star \star}$ \\
Acetabular depth $(\mathrm{mm})$ & $27.10 \pm 2.60(23.89-33.30)$ & $15.06 \pm 2.95(9.10-21.90)^{\star \star}$ \\
Acetabular volume $\left(\mathrm{mm}^{3}\right)$ & $35,510.44 \pm 7,809.78(27,341.98-48,034.93)$ & $7,549.46 \pm 2,535.61(5,547.43-13,757.24)^{\star *}$ \\
Cup size $(\mathrm{mm})$ & $52.67 \pm 2.39(50-56)$ & $44.67 \pm 1.44(44-48)^{\star \star}$ \\
Cup center height $(\mathrm{mm})$ & $16.29 \pm 1.44(13.80-19.30)$ & $13.32 \pm 1.70(8.67-16.25)^{\star \star}$ \\
Cup-CE $\left(^{\circ}\right)$ & $46.10 \pm 5.53(31.74-55.69)$ & $23.44 \pm 7.62(8.20-40.13)^{\star \star}$ \\
Cup-Sharp $\left(^{\circ}\right)$ & $35.43 \pm 4.07(27.39-46.84)$ & $49.01 \pm 5.60(34.95-59.51)^{\star *}$ \\
Acetabular anteversion angle $\left(^{\circ}\right)$ & $20.46 \pm 7.48(7.13-37.68)$ & $34.62 \pm 6.37(20.74-50.14)^{\star *}$ \\
Medial thickness $(\mathrm{mm})$ & $3.58 \pm 1.22(2.21-6.66)$ & $6.30 \pm 2.77(2.53-14.31)^{\star \star}$ \\
\hline
\end{tabular}

Values are presented as the mean and the standard deviation, with ranges in parentheses. ${ }^{* *}, \mathrm{P}<0.01$ when compared with the control group. DDH, developmental dysplasia of the hip.

Table 3 Measurements of 2D/3D coverage parameters

\begin{tabular}{lcc}
\hline Parameters & Normal $(n=36)$ & Crowe IV DDH $(\mathrm{n}=42)$ \\
\hline A-ASA $\left(^{\circ}\right)$ & $73.32 \pm 8.16(56.24-100.23)$ & $55.99 \pm 8.72(20.63-71.42)^{\star \star}$ \\
AS-ASA $\left(^{\circ}\right)$ & $119.05 \pm 6.42(107.35-132.24)$ & $84.77 \pm 11.83(64.82-112.17)^{\star \star}$ \\
S-ASA $\left(^{\circ}\right)$ & $135.93 \pm 5.42(121.26-144.69)$ & $113.44 \pm 7.62(98.20-130.13)^{\star \star}$ \\
PS-ASA $\left(^{\circ}\right)$ & $133.18 \pm 6.47(115.85-144.58)$ & $115.10 \pm 9.09(94.49-135.56)^{\star \star}$ \\
P-ASA $\left(^{\circ}\right)$ & $108.88 \pm 9.45(72.50-126.66)$ & $107.40 \pm 12.10(87.16-134.83)$ \\
Coverage ratio $(\%)$ & $94.51 \pm 2.32(88.39-98.60)$ & $79.89 \pm 4.82(72.25-89.84)^{\star \star}$ \\
\hline
\end{tabular}

Values are presented as the mean and the standard deviation, with ranges in parentheses. ${ }^{* *}, \mathrm{P}<0.01$ when compared with the control group. DDH, developmental dysplasia of the hip; ASA, acetabular sector angles.

more anteverted and abductive, with smaller Cup-CE and larger Cup-Sharp angles. Medially, the acetabular wall was significantly thicker in Crowe IV hips than in normal hips $(6.30 \pm 2.77$ vs. $3.58 \pm 1.22 \mathrm{~mm})$.

\section{Acetabular coverage angles and component coverage ratio}

As described in previous studies (7), the abnormally distributed bone stock was mainly located at the posterior rim of the true acetabulum. The coverage angles in Crowe IV hips were significantly smaller in the other 4 directions, except the posterior direction $(t=-0.628 ; \mathrm{P}=0.549)$. Further, the component coverage ratio was also significantly lower in the dysplastic hips (79.89\% vs. 94.51\%) (Table 3).
According to the implanted level, the acetabular medial thickness increased with the V-HCC in Crowe IV hips. However, the coverage ratio increased to the peak values at $12-21 \mathrm{~mm}$ above the DPCN. When the V-HCC was $>30 \mathrm{~mm}$, the host bone coverage ratio could not reach $70 \%$. When implantation was performed at the level of the true acetabulum, the coverage ratio was greatest, with a mean height of $13.32 \mathrm{~mm}$ (Table 4).

\section{Location analysis of the acetabular component}

For the coordinate system, the acetabular component center was defined as the origin $(\mathrm{O})$, and the projection of the ipsilateral ASIS was defined as the direction of the $y$-axis. 
Table 4 Medial thickness and coverage ratio with increased V-HCC in Crowe IV developmental dysplasia of the hip

\begin{tabular}{lcc}
\hline V-HCC $(\mathrm{mm})$ & $\begin{array}{c}\text { Medial thickness } \\
(\mathrm{mm})\end{array}$ & $\begin{array}{c}\text { Coverage ratio } \\
(\%)\end{array}$ \\
\hline $\begin{array}{l}\text { Implantation at true } \\
\text { acetabulum }(13.32 \pm 1.70)\end{array}$ & $6.30 \pm 2.77$ & $79.89 \pm 4.82$ \\
9 & $6.00 \pm 2.46$ & $72.58 \pm 5.92$ \\
12 & $6.15 \pm 2.71$ & $76.13 \pm 5.60$ \\
15 & $6.30 \pm 2.73$ & $78.99 \pm 5.50$ \\
18 & $6.33 \pm 2.50$ & $77.82 \pm 6.73$ \\
21 & $7.02 \pm 3.15$ & $75.69 \pm 6.76$ \\
24 & $9.67 \pm 5.56$ & $73.56 \pm 7.10$ \\
27 & $12.82 \pm 6.02$ & $71.24 \pm 6.82$ \\
30 & $17.87 \pm 6.58$ & $70.23 \pm 7.33$ \\
33 & $21.83 \pm 4.60$ & $69.65 \pm 7.22$ \\
36 & $23.53 \pm 3.39$ & $68.85 \pm 7.35$ \\
39 & $24.05 \pm 3.12$ & $68.17 \pm 7.90$ \\
\hline
\end{tabular}

Values are presented as the mean and the standard deviation. V-HCC, vertical height of the cup center.

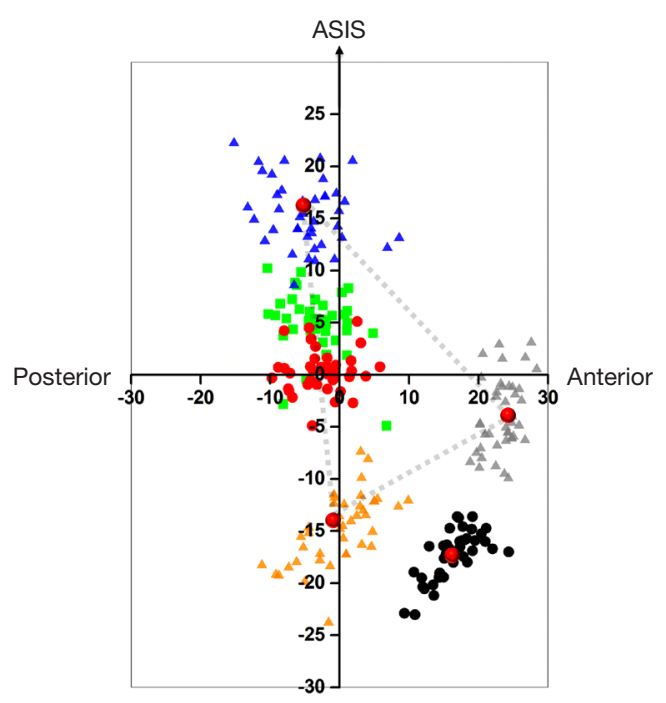

Figure 5 Projections of the bony landmarks to the component opening plane in the reference of the right hip. $\mathrm{O}$ is the component center. Blue, grey, and orange triangles represent projections of points T, A, and P, respectively. Black dots represent projections of DPCN. Red and green dots represent projections of midpoint $\left(\mathrm{O}_{1}\right)$ and upper one-third point $\left(\mathrm{O}_{2}\right)$ of posterior side in the projected triangle (grey dotted line). DPCN, distal part of the cotyloid notch.
The projections of 3 extreme landmarks of the acetabular rim were also depicted in reference to the right hip (Figure 5). As illustrated, the component center was positioned posteriorly and superiorly to the center of the triangular acetabulum. Therefore, the midpoint $\left(\mathrm{O}_{1}\right)$ and upper one-third point $\left(\mathrm{O}_{2}\right)$ of the posterior side in the projected triangle were digitized. Statistically, the mean distance from the origin towards $\mathrm{O}_{1}$ was significantly shorter than $\mathrm{O}_{2}(t=-11.22 ; \mathrm{P}<0.01)$ (Table 5). There were 29 hips $(69 \%)$ with a distance of $\mathrm{OO}_{1}$ within $5 \mathrm{~mm}$, and the distance was within $10 \mathrm{~mm}$ in all hips (100\%). The distance of $\mathrm{OO}_{2}$ was within $5 \mathrm{~mm}$ in 7 hips $(16 \%)$ and within $10 \mathrm{~mm}$ in 35 hips (83\%).

\section{Reproducibility}

The ICC results of the intraobserver and interobserver reliabilities for all the measurement indices, as evaluated by the one-way random effects model, ranged from 0.92 to 0.97 and 0.88 to 0.95 , respectively. Post-hoc power analysis showed a power $>0.94$ for detecting a significant difference.

\section{Discussion}

When performing a primary THA for a patient with Crowe IV DDH, the acetabular reconstruction, in particular, presents considerable technical challenges to orthopedic surgeons. Further, component revision in highly dislocated hips has a significant association with the acetabular osteolysis, cup instability, and severe polyethylene wear both in mid-term and long-term clinical studies (9,21-23). During the prior study, we noted that the acetabular anatomy and bone stock distribution were relatively constant in patients with Crowe IV dysplastic dislocation. Several studies have reported that $3 \mathrm{D}$ simulation can not only provide highresolution visualization of morphological changes in skeletal disorders, but can also predict the postoperative rotation center and the orientation of prosthesis with high validity and accuracy $(4,13,16)$. In the present study, we presented the anatomical size, orientation angles, and 2D/3D coverage indices of the true acetabulum quantitatively. In addition, the feasibility and optimal placement of a standard-sized cup for acetabular reconstruction was also determined in Crowe IV hips.

Compared with other Crowe types, the true acetabulum in Crowe IV is thoroughly separated from the dislocated 
Table 5 Location analysis of the acetabular component

\begin{tabular}{lcc}
\hline Parameters & $\mathrm{O}_{1}$ & $\mathrm{O}_{2}$ \\
\hline X-axis $(\mathrm{mm})$ & $-2.53 \pm 3.61(-9.76$ to 5.81$)$ & $-3.25 \pm 3.85(-10.40 \text { to } 6.73)^{\star \star}$ \\
Y-axis $(\mathrm{mm})$ & $0.38 \pm 2.00(-4.90$ to 5.09$)$ & $5.04 \pm 2.88(-4.89 \text { to } 10.22)^{\star \star}$ \\
Distance $(\mathrm{mm})$ & $4.12 \pm 2.53(0.58$ to 9.76$)$ & $7.18 \pm 2.67(2.14 \text { to } 14.58)^{\star \star}$ \\
\hline
\end{tabular}

Values are presented as the mean and the standard deviation, with range in parentheses. $\mathrm{O}_{1}$ and $\mathrm{O}_{2}$ were defined as the midpoint and upper one-third point of the posterior side in projected acetabular triangle. ${ }^{*}, \mathrm{P}<0.05 ;{ }^{* *}, \mathrm{P}<0.01$.

femoral head, resulting in significant dysplasia and deformation. A Crowe IV acetabulum presents as a narrow, shallow, and low-volume socket, consistent with a previous description (7). Acetabular volume in the triangular acetabulum is less than one-third that of a normal acetabulum, resulting in a significantly smaller component size and lower implanted center height. Due to the lack of articular contact, the Harris fossa is covered by few osteophytes and easy to identify. However, correct reorganization of the inferior edge of the true acetabulum is of particular importance for component implantation intraoperatively $(14,24)$.

After simulated implantation, the Crowe IV acetabulum tended to be sharply abductive, with a smaller Cup-CE and larger Cup-Sharp. In a 5-year follow-up study, Fujii et al. (25) suggested that Cup-CE angle by host bone should be greater than $0^{\circ}$ for satisfactory bony fixation. In the present study, the Cup-CE was $23.44^{\circ} \pm 7.62^{\circ}$ according to the lateral edge of the host bone, which indicates that there is no need for structural bone graft in most Crowe IV acetabular reconstructions $(9,26)$. The Cup-Sharp angle is also considered an important parameter to reflect the acetabular inclination and bony coverage during acetabular reconstruction. Although the Cup-Sharp angle was larger in the Crowe IV group than in the control group, it was still smaller than that in the Crowe I and II/III hips in our previous study (4). Compared with normal acetabulum, the dysplastic acetabulum had more excessive anteversion and thicker medial wall, which was also similar to our previous report (4). In the present study, the component was uniformly oriented at 20 anteversion and tangent to the inner cortex of the medial acetabular wall. Surgeons generally apply the technique of combined anteversion and acetabular cotyloplasty for better bony coverage. Although there was relatively sufficient bone stock medially, spongy bone condition and inadequate bone stock in the anteroposterior direction should also be taken into consideration during acetabular reaming.
Quantitative coverage analysis suggested that segmental deficiency was apparent at the entire acetabular rim, except for the posterior direction. In comparison, the most severe dysplastic bone stock was in the anterosuperior direction, whose sector angle decreased more than $35^{\circ}$ compared with normal hips. Due to great anteversion, there was no significant difference in the posterior coverage angles between the Crowe IV and control groups. Further, posterosuperior deficiency was less severe than that in the anterosuperior and superior directions, which is consistent with the description of abnormal bone distribution in previous studies $(5,7)$. Overall, $3 \mathrm{D}$ coverage can achieve $79.89 \%$ coverage by host bone with standard-sized cup implantation. Through 3D planning and clinical practice, we noted that relatively abundant bone stock in the posterosuperior and posterior acetabulum can provide considerable bone contact to accommodate a standard-sized cup with satisfactory coverage.

In terms of the limitation of the thin polyethylene liner and extremely small femoral head, a standard-sized component with ceramic-on-ceramic bearing has been recommended in recent studies $(10,12,13)$. According to the quantitative evaluation at different implantation levels, our findings indicated that standard-sized component coverage was $>75 \%$ when $\mathrm{V}-\mathrm{HCC}=12-21 \mathrm{~mm}$, increasing to peak values at the level of $13.32 \mathrm{~mm}$. When the V-HCC was $>30 \mathrm{~mm}$, the host bone coverage ratio did not reach $70 \%$. Various studies have focused on the minimum coverage provided by native host bone for securing longterm cup fixation without additional support. Garvin et al. (27) suggested that structural bone graft is not needed when host bone coverage is above $75 \%$ of the acetabular component. Jasty et al. (28) suggested that the cut-off value of structural bone grafting is $70 \%$, which was supported by Anderson and Harris, as well as by Shinar and Harris $(8,29)$. Considering the coverage variations, satisfactory acetabular coverage in Crowe IV hips should be performed cautiously by anatomical reconstruction, rather than high hip center 

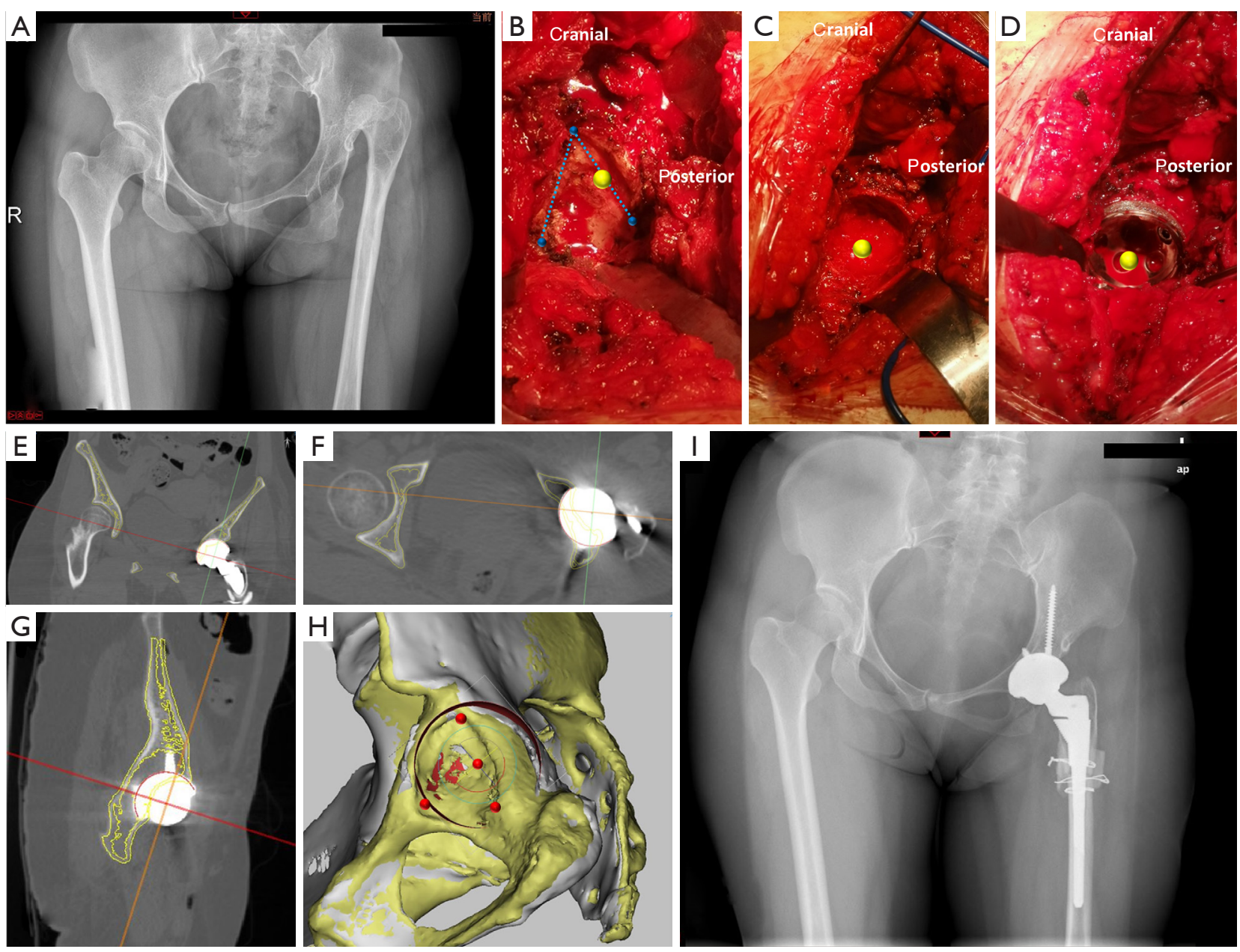

Figure 6 Perioperative radiographs and intraoperative photographs of a 38-year-old female patient with unilateral Crowe type-IV developmental dysplasia of the hip. (A) Preoperative anteroposterior pelvic view. (B) Intraoperative images of dysplastic acetabulum (blue bony reference points and dotted lines) and reaming center (yellow point). (C) Intraoperative images of prepared acetabulum. (D) Intraoperative images of component implantation. (E-H) Based on the postoperative CT, 3D pelvis model (white) and actual component was reconstructed. Preoperative simulated implantation (combination of yellow pelvis model and red virtual component) was imported to verify the component center by matching the $3 \mathrm{D}$ reconstruction. (E) Coronal image. (F) Transverse image. (G) Sagittal image. (H) 3D reconstructive image. (I) Three-month postoperative anteroposterior pelvic view. CT, computed tomography; 3D, 3-dimensional.

placement in Crowe II/III hips (30,31).

A reliable bony landmark does not only provide accurate Identification for the surgeon but also avoids intraoperative complications (32). Based on the component opening plane, useful bony landmarks were projected by $3 \mathrm{D}$ simulation. Our data showed that the optimal component center was mainly located at the midpoint between the superolateral and posteroinferior points in the coordinate system, demonstrating good feasibility and accuracy intraoperatively (Figure 6). The average distance between the selected point and component center was only
$4.12 \mathrm{~mm}: 2.53 \mathrm{~mm}$ horizontally and 0.38 vertically. Xu et al. (13) suggested that the component center in Crowe IV should be placed posterosuperiorly, described to be $4.55 \mathrm{~mm}$ superior and $4.37 \mathrm{~mm}$ posterior to the center of true acetabular circumcircle. Yoshitani et al. (5) revealed that the ideal center position is located at the upper third point of the posterior bony wall. In contrast, the mean distance in our data was less and variation range was within $10 \mathrm{~mm}$ in all hips. Moreover, the extreme poles of the acetabulum had better recognition and lower risk of abrasion and impingement than the acetabular wall. 


\section{Limitations}

The limitations of our study should be noted. First, the sample size was relatively small. However, Crowe IV hips are uncommon, and the results of the statistical analysis indicated reliable reproducibility. Second, demographic differences were present between the DDH and control groups. However, the recruitment of bilaterally affected hips to the DDH group restricted the selection of contralateral acetabulum as controls. Accordingly, a body mass index-matched control group would ensure more convincing results in future clinical studies. Third, all the components were oriented at $40^{\circ}$ abduction and $20^{\circ}$ anteversion, while implantation could be adjusted to be more abductive and anteverted individually during the actual operation. The orientation was decided according to the relative morphological studies. In the present study, unified implantation provided reliable and repeatable data, with satisfactory coverage ratio. As the actual weightbearing area was difficult to define in the $3 \mathrm{D}$ environment, we chose to weigh the uncovered portions equally.

\section{Conclusions}

The distinctively triangular acetabulum in Crowe IV DDH was found to be more anteverted and abductive, with a significantly narrow and low-volume opening. The most satisfactory coverage was achieved at the level of the true acetabulum by using standard-sized cup, and the prominent deficiency was mainly located in the anterosuperior and superior directions. Through simulated implantation, the optimal component center was determined as the midpoint between the superolateral and posteroinferior points. Adequate preoperative planning and cautious reaming should be carefully considered to avoid small-sized components in Crowe IV hips.

\section{Acknowledgments}

Funding: This study was supported by the Technological Research Fund of Guangdong Provincial People's Hospital (No. 2018bq11), Medical Science and Technology Research Fund of Guangdong Province (Nos. A2020026, A2019150), Natural Science Foundation of Guangdong Province (No. 2021A1515011008), Foundation of Administration of Traditional Chinese Medicine of Guangdong Province (No. 20211002), and Program of Science and Technology of Guangzhou (Nos. 202102020959, 201904010424).

\section{Footnote}

Reporting Checklist: The authors have completed the MDAR checklist. Available at https://qims.amegroups.com/article/ view/10.21037/qims-21-803/rc

Conflicts of Interest: All authors have completed the ICMJE uniform disclosure form (available at https://qims. amegroups.com/article/view/10.21037/qims-21-803/coif). The authors have no conflicts of interest to declare.

Ethical Statement: The authors are accountable for all aspects of the work in ensuring that questions related to the accuracy or integrity of any part of the work are appropriately investigated and resolved. The study was conducted in accordance with the Declaration of Helsinki (as revised in 2013). This study was approved by the Institutional Review Board of Guangdong Provincial People's Hospital (No. 2019528HR1), and informed consent was obtained in writing from all the individual participants included in the study.

Open Access Statement: This is an Open Access article distributed in accordance with the Creative Commons Attribution-NonCommercial-NoDerivs 4.0 International License (CC BY-NC-ND 4.0), which permits the noncommercial replication and distribution of the article with the strict proviso that no changes or edits are made and the original work is properly cited (including links to both the formal publication through the relevant DOI and the license). See: https://creativecommons.org/licenses/by-nc-nd/4.0/.

\section{References}

1. Argenson JN, Flecher X, Parratte S, Aubaniac JM. Anatomy of the dysplastic hip and consequences for total hip arthroplasty. Clin Orthop Relat Res 2007;465:40-5.

2. Sanchez-Sotelo J, Berry DJ, Trousdale RT, Cabanela ME. Surgical treatment of developmental dysplasia of the hip in adults: II. Arthroplasty options. J Am Acad Orthop Surg 2002;10:334-44.

3. Watts CD, Abdel MP, Hanssen AD, Pagnano MW. Anatomic Hip Center Decreases Aseptic Loosening Rates After Total Hip Arthroplasty with Cement in Patients with Crowe Type-II Dysplasia: A Concise Follow-up Report at a Mean of Thirty-six Years. J Bone Joint Surg Am 2016;98:910-5.

4. Yang Y, Zuo J, Liu T, Xiao J, Liu S, Gao Z. Morphological 
Analysis of True Acetabulum in Hip Dysplasia (Crowe Classes I-IV) Via 3-D Implantation Simulation. J Bone Joint Surg Am 2017;99:e92.

5. Yoshitani J, Kabata T, Kajino Y, Ueno T, Ueoka K, Nakamura T, Tsuchiya H. Morphometric geometrical analysis to determine the centre of the acetabular component placement in Crowe type IV hips undergoing total hip arthroplasty. Bone Joint J 2019;101-B:189-97.

6. Zeng Y, Lai OJ, Shen B, Yang J, Zhou ZK, Kang PD, Pei FX, Zhou X. Three-dimensional computerized preoperative planning of total hip arthroplasty with highriding dislocation developmental dysplasia of the hip. Orthop Surg 2014;6:95-102.

7. Hartofilakidis G, Stamos K, Karachalios T. Treatment of high dislocation of the hip in adults with total hip arthroplasty. Operative technique and long-term clinical results. J Bone Joint Surg Am 1998;80:510-7.

8. Anderson MJ, Harris WH. Total hip arthroplasty with insertion of the acetabular component without cement in hips with total congenital dislocation or marked congenital dysplasia. J Bone Joint Surg Am 1999;81:347-54.

9. Mu W, Yang D, Xu B, Mamtimin A, Guo W, Cao L. Midterm Outcome of Cementless Total Hip Arthroplasty in Crowe IV-Hartofilakidis Type III Developmental Dysplasia of the Hip. J Arthroplasty 2016;31:668-75.

10. Ding ZC, Zeng WN, Mou P, Liang ZM, Wang D, Zhou ZK. Risk of Dislocation After Total Hip Arthroplasty in Patients with Crowe Type IV Developmental Dysplasia of the Hip. Orthop Surg 2020;12:589-600.

11. Sen C, Bilsel K, Elmadag M, Gunes T, Saygi B. Acetabuloplasty at the anatomic centre for treating Crowe class III and IV developmental hip dysplasia: a case series. Hip Int 2016;26:360-6.

12. Zhou Y, Sun C, Wang Y. New Method Addressing the Problem of Using Ceramic-on-Ceramic Bearing in Too Small Acetabulum of High-Riding DDH Patients with THA. Seminars in Arthroplasty 2012;23:226-31.

13. Xu J, Xu C, Mao Y, Zhang J, Li H, Zhu Z. Posterosuperior Placement of a Standard-Sized Cup at the True Acetabulum in Acetabular Reconstruction of Developmental Dysplasia of the Hip With High Dislocation. J Arthroplasty 2016;31:1233-9.

14. Zhang H, Zhou J, Guan J, Ding H, Wang Z, Dong Q. How to restore rotation center in total hip arthroplasty for developmental dysplasia of the hip by recognizing the pathomorphology of acetabulum and Harris fossa? J Orthop Surg Res 2019;14:339.

15. Lewinnek GE, Lewis JL, Tarr R, Compere CL,
Zimmerman JR. Dislocations after total hip-replacement arthroplasties. J Bone Joint Surg Am 1978;60:217-20.

16. Sariali E, Boukhelifa N, Catonne Y, Pascal Moussellard H. Comparison of Three-Dimensional Planning-Assisted and Conventional Acetabular Cup Positioning in Total Hip Arthroplasty: A Randomized Controlled Trial. J Bone Joint Surg Am 2016;98:108-16.

17. Vandenbussche E, Saffarini M, Taillieu F, Mutschler C. The asymmetric profile of the acetabulum. Clin Orthop Relat Res 2008;466:417-23.

18. Jóźwiak M, Rychlik M, Musielak B, Chen BP, Idzior M, Grzegorzewski A. An accurate method of radiological assessment of acetabular volume and orientation in computed tomography spatial reconstruction. BMC Musculoskelet Disord 2015;16:42.

19. Lee C, Jang J, Kim HW, Kim YS, Kim Y. Threedimensional analysis of acetabular orientation using a semiautomated algorithm. Comput Assist Surg (Abingdon) 2019;24:18-25.

20. Faul F, Erdfelder E, Lang AG, Buchner A. G*Power 3: a flexible statistical power analysis program for the social, behavioral, and biomedical sciences. Behav Res Methods 2007;39:175-91.

21. Imbuldeniya AM, Walter WL, Zicat BA, Walter WK. Cementless total hip replacement without femoral osteotomy in patients with severe developmental dysplasia of the hip: minimum 15-year clinical and radiological results. Bone Joint J 2014;96-B:1449-54.

22. Sofu H, S Ahin V, Gürsu S, Yildirim T, Issin A, Koçkara N. Cementless total hip arthroplasty in patients with Crowe type-4 developmental dysplasia. Hip Int 2013;23:472-7.

23. Grammatopoulos G, Pandit H, Glyn-Jones S, McLardySmith P, Gundle R, Whitwell D, Gill HS, Murray DW. Optimal acetabular orientation for hip resurfacing. J Bone Joint Surg Br 2010;92:1072-8.

24. Hartofilakidis G, Babis GC, Lampropoulou-Adamidou $\mathrm{K}$, Vlamis J. Results of total hip arthroplasty differ in subtypes of high dislocation. Clin Orthop Relat Res 2013;471:2972-9.

25. Fujii M, Nakashima Y, Nakamura T, Ito Y, Hara T. Minimum Lateral Bone Coverage Required for Securing Fixation of Cementless Acetabular Components in Hip Dysplasia. Biomed Res Int 2017;2017:4937151.

26. Rasi AM, Kazemian G, Khak M, Zarei R. Shortening subtrochanteric osteotomy and cup placement at true acetabulum in total hip arthroplasty of Crowe III-IV developmental dysplasia: results of midterm follow-up. Eur J Orthop Surg Traumatol 2018;28:923-30. 
27. Garvin KL, Bowen MK, Salvati EA, Ranawat CS. Longterm results of total hip arthroplasty in congenital dislocation and dysplasia of the hip. A follow-up note. J Bone Joint Surg Am 1991;73:1348-54.

28. Jasty M, Anderson MJ, Harris WH. Total hip replacement for developmental dysplasia of the hip. Clin Orthop Relat Res 1995;(311):40-5.

29. Shinar AA, Harris WH. Bulk structural autogenous grafts and allografts for reconstruction of the acetabulum in total hip arthroplasty. Sixteen-year-average follow-up. J Bone Joint Surg Am 1997;79:159-68.

30. Zheng LL, Lin YY, Zhang XY, Ling QH, Liao WM, $\mathrm{Wu} \mathrm{PH}$. Best bone of acetabulum for cup component

Cite this article as: Yang Y, Ma Y, Li Q, Lin B, Dong H, Zheng Q. Three-dimensional morphological analysis of true acetabulum in Crowe type IV hip dysplasia via standardsized cup-simulated implantation. Quant Imaging Med Surg 2022;12(5):2904-2916. doi: 10.21037/qims-21-803 placement in Crowe types I to III dysplastic hips: a computer simulation study. Chin Med J (Engl) 2019;132:2820-6.

31. Chen M, Luo ZL, Wu KR, Zhang XQ, Ling XD, Shang XF. Cementless Total Hip Arthroplasty With a High Hip Center for Hartofilakidis Type B Developmental Dysplasia of the Hip: Results of Midterm Follow-Up. J Arthroplasty 2016;31:1027-34.

32. Shimodaira H, Tensho K, Akaoka Y, Koyama S, Maruyama M, Kato H, Saito N. The Acetabular Fossa May Not Be Located at the True Center of the Acetabulum: A Detailed Analysis Using Preoperative CT Images. J Bone Joint Surg Am 2018;100:e27. 